\title{
Prognostic factors for renal allograft survival in patients with immunoglobulin A nephropathy: A case control study
}

\author{
JUN-QI GUO $^{1 *}$, BAO-LIN SONG ${ }^{2 *}$, ZHI-XIAN WU $^{1 *}$, WEI-ZHEN WU ${ }^{1 *}$, LIU-TAO LUO $^{1}$, XIAO-WEN CHEN $^{1}$, \\ FU-QIANG HE ${ }^{1}$, ZHI-YONG ZHENG ${ }^{1}$, SHUN-LIANG YANG ${ }^{1}$ and JIAN-MING TAN ${ }^{1}$ \\ ${ }^{1}$ Organ Transplant Institute, Fuzhou General Hospital, Fujian Medical University and Fujian Key Laboratory of \\ Transplant Biology, Fuzhou, Fujian 350025; ${ }^{2}$ Department of Urology, Jiaxing Traditional Chinese Medical Hospital, \\ Jiaxing, Zhejiang 314000, P.R. China
}

Received June 25, 2013; Accepted January 31, 2014

DOI: $10.3892 / \mathrm{mmr} .2014 .1954$

\begin{abstract}
The renal allograft survival rates of patients with immunoglobulin A nephropathy (IgAN), and patients with or without other glomerular diseases, have yet to be fully elucidated. In this study, the clinicopathological factors associated with long-term allograft survival for the prognosis of renal allograft recipients with IgAN were examined. All patients enrolled in this study were diagnosed with IgAN following clinical and pathological examinations. Patients underwent renal graft biopsy and were hospitalized at the Fuzhou General Hospital between June, 2004 and December, 2010. Common demographic and clinical indicators were recorded in patients who had graft loss and in those who had functional renal grafts. Forty-two of the 202 biopsy specimens $(20.8 \%)$ met the diagnostic criteria for IgAN and were divided into two groups, the graft loss group $(n=17)$ and the functional graft group $(n=25)$. Patients were followed up for 1-257 months after kidney transplantation. The mean patient age was $40.6 \pm 9.3$ years at the time of renal graft biopsy. Examination results indicated concomitant proteinuria and hematuria in 25 patients $(59.5 \%)$ and proteinuria alone in six patients (14.3\%). Graft loss occurred in 17 patients during the follow-up period. Comparison of the graft loss and the functional graft groups indicated that patients in the graft loss group were more likely to have proteinuria $(\mathrm{P}=0.047)$, high creatinine levels at the time of biopsy $(\mathrm{P}=0.009)$, low glomerular filtration rates $(\mathrm{P}=0.013)$, low serum total protein $(\mathrm{P}=0.01)$, a high Banff score $(\mathrm{P}=0.001)$, extensive glomerulosclerosis $(\mathrm{P}=0.002)$, a greater likelihood of cres-
\end{abstract}

Correspondence to: Dr Jian-Ming Tan, Organ Transplant Institute, Fuzhou General Hospital, Fujian Medical University and Fujian Key Laboratory of Transplant Biology, 156 Xi Er Huang Road, Fuzhou, Fujian 350025, P.R. China

E-mail: guojunq2006@163.com

*Contributed equally

Key words: kidney graft, immunoglobulin A nephropathy, prognostic factors cent formation $(\mathrm{P}=0.01)$, severe tubular atrophy $(\mathrm{P}=0.013)$ and more extensive interstitial fibrosis $(\mathrm{P}=0.033)$. However, the two groups showed no significant differences in blood pressure, hematuria, BUN, UA, Hb, TG and CHO levels. The allograft survival rate of patients with IgAN was identified to be similar to that of patients with and without other glomerular diseases.

\section{Introduction}

Immunoglobulin A nephropathy (IgAN) is a renal disorder, which is characterized by symptomatic and pathological changes caused by diffuse deposition of IgA or IgA-containing immune complexes in the glomerular mesangial area and capillary loops. IgAN is increasingly common in males than in females and has been observed in a high percentage of renal biopsies in Asia. Bi (1) and Li (2) identified that IgAN accounts for $26-34 \%$ of all primary glomerular diseases in China and is one of the main causes of end stage renal disease (ESRD).

Kidney transplantation is considered the optimal therapeutic strategy for ESRD. Berger et al (3) were the first to report recurrent $\operatorname{IgAN}$ in kidney allograft recipients and recurrent $\operatorname{Ig} \mathrm{A}$ deposition in the mesangial area of kidney allograft recipients. Additionally, they found that recurrent IgAN in kidney allograft recipients had a relatively benign clinical course. Earlier studies have shown that recurrent IgAN does not have a negative impact on the clinical course of kidney transplantation (4). However, studies published in the 1990's that employed longer follow-up periods reported that recurrent $\operatorname{Ig} \mathrm{AN}$ was a major cause of graft loss $(5,6)$. In particular, recurrent IgAN was observed in $20-60 \%$ of patients following transplantation and certain transplant recipients experienced de novo $\operatorname{Ig} \mathrm{AN}$ (7-11).

At present, the prognostic factors associated with recurrent IgAN in kidney allograft recipients in China remain to be fully elucidated. Although there have been numerous Western studies of the incidence rate and risk factors of recurrent $\operatorname{IgAN}$ in kidney allograft recipients, there are few studies focusing on the prognostic factors of recurrent IgAN following kidney transplantation $(12,13)$. In the present study, the prognostic factors associated with recurrent $\operatorname{IgAN}$ in 
kidney allograft recipients from a single institution in China were investigated.

\section{Materials and methods}

Clinical data. Patients undergoing renal graft biopsy $(\mathrm{n}=197)$ who were hospitalized at the Organ Transplant Center of the Fuzhou General Hospital (Fuzhou, China) between June, 2004 and December, 2010 were screened. A total of 202 kidney allograft biopsy specimens were obtained. Forty-two patients (20.8\%, including 31 males and 11 females) met the diagnostic criteria for IgAN following clinical and pathological examinations. Thirty-eight cases received one transplantation and four cases received two transplantations. There were 41 cadaveric donor kidney transplants and 1 live-donor kidney transplant. Follow-up was every three months and the final follow-up occured on August 31, 2012. The study was approved by the Ethics Committee of the Organ Transplant Institute, Fuzhou General Hospital, Fuzhou, China (Review board IRB00006161) and written informed consent was obtained from the patients.

The 42 patients who met the diagnostic criteria for IgAN were divided into two groups, the graft loss group $(n=17)$ and the functional graft group $(n=25)$. The graft loss group included patients with graft dysfunction, those who required resumption of dialysis or a second transplant. Pathological diagnosis was based on conventional hematoxylin and eosin, periodic acid-schiff, Masson and immunohistochemical staining for IgG, IgM, IgA, C3d, C1q and C4d. The pathological changes of kidney grafts were observed by light microscopy (Leica Microsystems, Ltd., Hong Kong, China). The Banff 97 score of allograft nephropathy was calculated as described previously (14).

Clinical and pathological characteristics. The following demographic and clinical data were recorded: Age, gender, date of transplantation, requirement for repeat transplantation, cadaveric or live donor transplantation, date of IgAN onset, renal graft biopsy and graft dysfunction, height, weight, blood pressure, pulse, urine output at the time of biopsy, edema, gross hematuria and immunosuppressant regimen and dose. The following clinical indicators were measured 1 week prior to renal graft biopsy: Routine urine test, 24-h urinary protein levels for patients with proteinuria, serum creatinine ( $\mathrm{SCr}$ ), blood urea nitrogen (BUN), uric acid (UA), glomerular filtration rate, hemoglobin $(\mathrm{Hb})$, triglycerides $(\mathrm{TG})$, total cholesterol (CHO), serum total protein (TPR) and serum albumin (Alb) levels. The following pathological characteristics were also recorded: Renal allograft nephropathy score, glomerular sclerosis, crescent formation, fibrinoid necrosis, tubular atrophy, interstitial fibrosis and arteriolopathy.

Statistical analysis. All data were recorded in a database. Quantitative data were compared using Student's t-test. If the data had non-normal distribution and/or heterogeneity of variance, a rank sum test was performed. Qualitative data were compared using the $\chi^{2}$ test. Renal graft survival rate was calculated using the Kaplan-Meier method, and differences in survival were examined with the log-rank test. All data were analyzed using SPSS software, version 13.0 (SPSS,
Inc., Chicago, IL, USA). $\mathrm{P}<0.05$ was considered to indicate a statistically significant difference.

\section{Results}

General characteristics of patients. Between June, 2004 and December, 2010, 197 patients underwent renal graft biopsy at the Organ Transplant Center, Fuzhou General Hospital and 202 kidney allograft biopsy specimens were obtained. Forty-two patients (20.8\%; including 31 males and 11 females) met the diagnostic criteria for IgAN. Thirty-eight patients underwent transplantation once and four patients underwent transplantation twice. Forty-one cases received cadaveric donor kidneys and one case received a kidney from a living donor. The examination results indicated the presence of concomitant proteinuria and hematuria in 25 patients $(59.5 \%)$ and proteinuria, but no hematuria in six patients $(14.3 \%)$. The concentration of urinary protein in these 31 patients indicated a mean of 3.6 $2.1 \mathrm{~g} / 24 \mathrm{~h}$ (range, 0.312-8.13 g/24 h). Analysis of all 42 biopsy specimens indicated the presence of 17.2 \pm 13.0 glomeruli. All specimens met the Banff 97 criteria for renal biopsy (14).

The graft loss and functional graft groups showed no statistically significant differences in gender, age, human leukocyte antigen (HLA) matching, immunosuppressant regimen and dose, blood pressure and urine output at the time of biopsy (Table I).

Analysis of clinical indicators. Analysis of the clinical data of the two groups indicated that patients in the graft loss group were more likely to exhibit proteinuria $(\mathrm{P}=0.014)$, higher $\mathrm{SCr}$ levels at the time of biopsy $(\mathrm{P}=0.009)$, lower epidermal growth factor receptor levels $(\mathrm{P}=0.013)$, lower TPR $(\mathrm{P}=0.01)$ and lower serum Alb levels $(\mathrm{P}=0.043)$ (Table II). However, the two groups showed no significant differences in hematuria, BUN, UA, Hb, TG and CHO levels (Table II).

Analysis of the pathological characteristics. Analysis of the pathological characteristics of the two groups indicated that patients in the graft loss group were more likely to have a high Banff score $(\mathrm{P}=0.001)$, severe glomerular sclerosis $(\mathrm{P}=0.002)$, increased crescent formation $(\mathrm{P}=0.01)$, greater tubular atrophy $(\mathrm{P}=0.013)$ and interstitial fibrosis $(\mathrm{P}=0.033)$ compared with those of the functional graft group (Table III).

Analysis of renal graft survival. During the same period (between June, 2004 and December, 2010), in 88 kidney allograft recipients who underwent renal graft biopsy at Fuzhou General Hospital, pathological examination confirmed the presence of other glomerular diseases, including acute diffuse proliferative glomerulonephritis, rapidly progressive glomerulonephritis, membranous glomerulonephritis, minimal change glomerulonephritis, focal segmental glomerulosclerosis, membranoproliferative glomerulonephritis, mesangial proliferative glomerulonephritis and chronic glomerulonephritis, based on the diagnostic criteria previously described (15). In addition, 36 of these patients demonstrated glomerular lesions, including renal interstitial lesions free of glomerular lesions, tubular lesions, arteriolopathy, acute and chronic rejection, and immunosuppressant toxicity. 
Table I. Demographic and clinical characteristics of enrolled patients with IgAN.

\begin{tabular}{|c|c|c|c|}
\hline Characteristics & Graft loss $(n=17)$ & Functional graft $(n=25)$ & P-value \\
\hline \multicolumn{4}{|l|}{ Gender } \\
\hline Males & 12 & 19 & \\
\hline Females & 5 & 6 & 0.733 \\
\hline Mean age (years) & $36.1 \pm 9.2$ & $38.1 \pm 4.3$ & 0.823 \\
\hline HLA mismatches & $2.29 \pm 0.69$ & $2.36 \pm 0.95$ & 0.881 \\
\hline HLA-A & $0.82 \pm 0.64$ & $0.8 \pm 0.82$ & 0.804 \\
\hline HLA-B & $0.71 \pm 0.69$ & $0.76 \pm 0.66$ & 0.777 \\
\hline HLA-DR & $0.76 \pm 0.66$ & $0.8 \pm 0.82$ & 0.989 \\
\hline \multicolumn{4}{|l|}{$\begin{array}{l}\text { Immunosuppressive drugs } \\
\text { at time of diagnosis }\end{array}$} \\
\hline Prednisolone (mg/day) & $8.6 \pm 2.0$ & $9.1 \pm 2.9$ & 0.603 \\
\hline $\mathrm{n}(\%)$ & $17(100)$ & $25(100)$ & 1 \\
\hline Ciclosporin (mg/day) & $225.0 \pm 35.4$ & $133.3 \pm 28.9$ & 0.076 \\
\hline $\mathrm{n}(\%)$ & $4(23.5)$ & $9(36.0)$ & 0.391 \\
\hline Tacrolimus (mg/day) & $2.2 \pm 1.1$ & $2.0 \pm 1.0$ & 0.606 \\
\hline $\mathrm{n}(\%)$ & $13(76.5)$ & $16(64.0)$ & 0.296 \\
\hline MMF (mg/day) & $892.9 \pm 349.3$ & $863.6 \pm 171.9$ & 0.961 \\
\hline $\mathrm{n}(\%)$ & $14(82.3)$ & $20(80.0)$ & 0.758 \\
\hline Bredinin (mg/day) & $87.5 \pm 17.7$ & $75 \pm 15.6$ & 0.317 \\
\hline $\mathrm{n}(\%)$ & $2(11.8)$ & $2(8.0)$ & 0.758 \\
\hline Azathioprine (mg/day) & 50 & 50 & 1.00 \\
\hline $\mathrm{n}(\%)$ & $1(5.9)$ & $3(12.0)$ & 0.758 \\
\hline \multicolumn{4}{|l|}{ Blood pressure (mmHg) } \\
\hline Systolic & $136.4 \pm 19.4$ & $135.8 \pm 18.5$ & 0.926 \\
\hline Diastolic & $86.4 \pm 12.4$ & $85.6 \pm 12.0$ & 0.833 \\
\hline Urine volume (ml/24 h) & $1872.7 \pm 392.7$ & $1742.5 \pm 595.0$ & 0.636 \\
\hline
\end{tabular}

IgAN, immunoglobulin A nephropathy; HLA, human leukocyte antigen; MMF, mycophenolate mofetil.

The cumulative graft survival rates of the three groups (patients with IgAN and patients with or without other glomerular diseases) over 10 years are shown in Fig. 1. The renal graft survival rate was calculated using the Kaplan-Meier method and identified no significant differences in the graft survival rates of the three groups over 10 years $(\mathrm{P}=0.122)$.

\section{Discussion}

The presence of $\operatorname{IgAN}$ in kidney allograft recipients may be due to recurrence of $\mathrm{IgAN}$, de novo $\mathrm{IgAN}$ or transmission of IgA deposits from the donor kidney. The majority of patients with kidney disease also presented with ESRD at medical examination and such patients are not suitable for biopsy. Thus, histopathological diagnosis of the primary disease is not available for the majority of kidney allograft recipients. In addition, time-zero biopsy data of donor grafts are generally not available. This renders it difficult or even impossible to differentiate recurrent $\operatorname{Ig} \mathrm{AN}$, de novo $\operatorname{Ig} \mathrm{AN}$ and donor-transmitted $\operatorname{Ig} \mathrm{AN}$. However, long term clinical observations at Fuzhou General Hospital indicated that the majority of donor kidneys with
IgA deposition at the baseline biopsy did not result in $\operatorname{Ig} \mathrm{A}$ deposition during postoperative allograft biopsy. In addition, Sanfilippo et al (16) showed that mesangial IgA deposition can quickly dissipate following transplantation. Thus, it was hypothesized that the majority of patients in the present study had recurrent $\operatorname{Ig} \mathrm{AN}$ or de novo $\operatorname{Ig} \mathrm{AN}$.

This study screened 197 hospitalized patients who underwent renal graft biopsy at the Organ Transplant Center of the Fuzhou General Hospital (Fuzhou, China) between June, 2004 and December, 2010. Of these, 42 patients (20.8\%) met the diagnostic criteria for $\operatorname{IgAN}$, indicating that $\operatorname{IgAN}$ was common among the kidney allograft recipients. Previous studies did not identify any clinical abnormalities in approximately one-third of patients with confirmed $\operatorname{IgAN}(5,17,18)$; therefore, it was suggested that there may have been increased cases of IgAN if renal allograft biopsies were performed for all kidney allograft recipients.

Abnormal routine urine test results were common in the renal allograft recipients with $\operatorname{IgAN}(83.3 \%)$, and microscopic examination indicated concomitant proteinuria and hematuria in 25 patients $(59.5 \%)$. Statistical analysis indi- 
Table II. Clinical indicators of patients in the graft loss and functional graft groups.

\begin{tabular}{lccc}
\hline Indicators & Graft loss $(\mathrm{n}=17)$ & Functional graft $(\mathrm{n}=25)$ & P-value \\
\hline Proteinuria, $\mathrm{n}(\%)$ & $16(94.1)$ & $15(60)$ & $0.014^{\mathrm{a}}$ \\
Hematuria, $\mathrm{n}(\%)$ & $13(76.5)$ & $16(64)$ & 0.391 \\
Proteinuria $(\mathrm{g} / 24 \mathrm{~h})$ & $4.1 \pm 2.4$ & $2.6 \pm 1.7$ & $0.047^{\mathrm{a}}$ \\
$\mathrm{SCr}(\mu \mathrm{mol} / \mathrm{l})$ & $373.6 \pm 267.5$ & $220.9 \pm 164.9$ & $0.009^{\mathrm{a}}$ \\
$\mathrm{BUN}(\mathrm{mmol} / \mathrm{l})$ & $16.9 \pm 9.5$ & $13.5 \pm 10.7$ & 0.081 \\
$\mathrm{UA}(\mathrm{mmol} / \mathrm{l})$ & $489.9 \pm 157.8$ & $480.7 \pm 133.8$ & 0.769 \\
eGFR $(\mathrm{ml} / \mathrm{min})$ & $29.3 \pm 18.2$ & $51.9 \pm 32.1$ & $0.013^{\mathrm{a}}$ \\
Hb $(\mathrm{g} / \mathrm{l})$ & $97.8 \pm 24.8$ & $107.9 \pm 21.8$ & 0.173 \\
TG $(\mathrm{mmol} / \mathrm{l})$ & $1.5 \pm 1.0$ & $1.5 \pm 0.7$ & 0.397 \\
CHO $(\mathrm{mmol} / \mathrm{l})$ & $5.8 \pm 3.5$ & $6.1 \pm 2.1$ & 0.182 \\
TPR $(\mathrm{g} / \mathrm{l})$ & $57.9 \pm 7.5$ & $66.5 \pm 10.9$ & $0.01^{\mathrm{a}}$ \\
Alb $(\mathrm{g} / \mathrm{l})$ & $34.3 \pm 5.1$ & $38.7 \pm 8.2$ & $0.043^{\mathrm{a}}$ \\
\hline
\end{tabular}

SCr, serum creatinine; BUN, blood urea nitrogen; UA, uric acid; eGFR, epidermal growth factor receptor; Hb, hemoglobin; TG, triglycerides; $\mathrm{CHO}$, total cholesterol; TPR, serum total protein; Alb, serum albumin. ${ }^{\mathrm{a}} \mathrm{P}<0.05$ compared to the function graft group.

Table III. Pathological characteristics of patients in the graft loss and functional graft groups.

\begin{tabular}{|c|c|c|c|}
\hline Characteristics & Graft loss $(n=17)$ & Functional graft $(n=25)$ & P-value \\
\hline Banff score (0-30) & $15.6 \pm 6.0$ & $8.8 \pm 4.6$ & $0.001^{\mathrm{a}}$ \\
\hline Concomitant CAN, n (\%) & $4(23.5)$ & $8(32.0)$ & 0.551 \\
\hline Concomitant AR, n (\%) & $1(5.9)$ & $2(8.0)$ & 0.794 \\
\hline Calcineurin inhibitor toxicity (\%) & $0(0.0)$ & $1(4.0)$ & 0.404 \\
\hline Glomerular sclerosis n (\%) & & & $0.002^{\mathrm{a}}$ \\
\hline $0 \%$ & $2(11.8)$ & $7(28.0)$ & \\
\hline $1-25 \%$ & $3(17.7)$ & $14(56.0)$ & \\
\hline$>25 \%$ & $12(70.6)$ & $4(16.0)$ & \\
\hline Crescent & $7(41.8)$ & $2(8.0)$ & $0.01^{\mathrm{a}}$ \\
\hline Tubular atrophy & & & $0.013^{\mathrm{a}}$ \\
\hline $0 \%$ & $0(0)$ & $3(12.0)$ & \\
\hline $1-25 \%$ & $2(11.8)$ & $11(44.0)$ & \\
\hline$>25 \%$ & $12(70.6)$ & $11(44.0)$ & \\
\hline Interstitial fibrosis & & & $0.033^{\mathrm{a}}$ \\
\hline $0-5 \%$ & $0(0)$ & $4(16.0)$ & \\
\hline $6-25 \%$ & $4(23.5)$ & $12(48.0)$ & \\
\hline$>25 \%$ & $13(76.5)$ & $10(40.0)$ & \\
\hline \multicolumn{4}{|l|}{ Vascular } \\
\hline Normal & 1 & 7 & \\
\hline Hyalinosis & 1 & 18 & 0.073 \\
\hline
\end{tabular}

${ }^{\text {aP }}<0.05$ compared with the functional graft group. CAN, chronic allograft nephropathy; AR, acute rejection.

cated that proteinuria, but not hematuria, was significantly associated with graft loss. The 24-h urinary protein level was also significantly greater in the graft loss group than that in the functional graft group. Moreover, the results indicated that serum TRP and Alb levels were significantly lower in the graft loss group compared with those in the functional graft group. Collectively, these results suggested that the substantial loss of protein in the urine is associated with poor graft outcome.

The results also indicated that higher $\mathrm{SCr}$ levels were significantly associated with graft loss. In particular, the mean SCr level was $373.6 \pm 267.5 \mu \mathrm{mol} / 1$ in the graft loss group and $220.9 \pm 164.9 \mu \mathrm{mol} / 1$ in the functional graft group at the time of biopsy. BUN and UA levels were not correlated with renal 


\section{Survival functions}

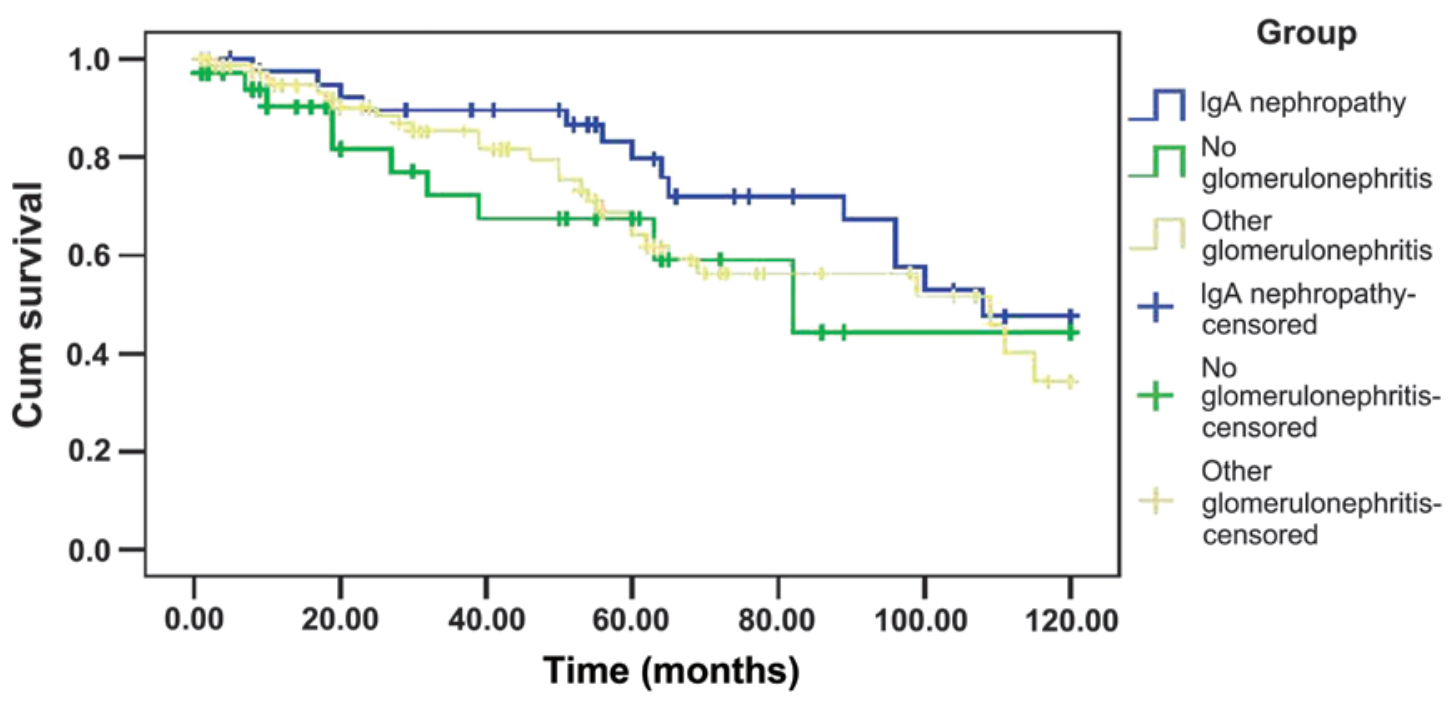

Figure 1. Cumulative renal graft survival rates in patients with $\operatorname{IgAN}$ and patients with or without other glomerular diseases over 10 years. IgAN, immunoglobulin A nephropathy.

graft prognosis. Concurrent with the results of the present study, Wang et al (19) demonstrated that patients with graft loss exhibited higher SCr levels at the time of renal graft biopsy and Kiattisunthorn et al (13) suggested that higher $\mathrm{SCr}$ and urinary protein levels were associated with poor graft function.

The present study did not identify a significant correlation between HLA matching and the prognosis of kidney allografts in patients with IgAN. However, previous studies have shown that females and patients with positive HLA matching were more prone to recurrent IgAN and had a lower 5-year graft survival rate $(20,21)$. In addition, Andresdottir et al $(22,23)$ investigated the graft survival of 1,207 patients with IgAN and 7,935 patients with no glomerular disease, and reported that the presence of the HLA-B8 and DR3 haplotype increased the risk of graft loss.

Namba et al (9) found that progressive graft loss in patients with recurrent IgAN was associated with the deterioration of renal graft function, hypertension and angiotensin converting enzyme inhibitor deficiency at the time of biopsy. However, the present study demonstrated no significant correlation between graft loss and hypertension, hypotension or pulse rate at the time of biopsy. The results also indicated that the levels of serum TG and $\mathrm{CHO}$ were not significantly associated with graft loss.

Increased crescent formation suggested a poor prognosis of the renal allograft. In the present study, a significant increase in the number of patients with crescent formation was observed in the graft loss group $(n=7 ; 41.8 \%)$ compared with the functional graft group $(\mathrm{n}=2 ; 8.0 \%)$. Tang et al $(24)$ proposed that $\operatorname{IgAN}$ with crescent formation is increasingly common in patients who received renal grafts or were diagnosed with primary glomerulonephritis in China. Such patients developed rapid progression of renal insufficiency, progressed to kidney dysfunction and ultimately required hemodialysis. Kowalewska et al (25) found that early renal dysfunction or resumption of dialysis occurred in approximately half of renal allograft recipients with IgAN who presented with crescent formation. Soler et al (21) showed that the 5-year graft survival rate was $71 \%$ in patients with crescentic glomerulonephritis who were diagnosed with IgA or HenochSchonlein purpura, but was $100 \%$ in patients with glomerular mesangial hyperplasia or focal segmental glomerulosclerosis.

Oka et al (26) showed that the pathological characteristics of IgAN in renal graft recipients were different from those in patients with IgAN who had not undergone transplantation. In particular, graft recipients with IgAN seldom displayed active damage, such as extracapillary proliferation, but often exhibited chronic damage, such as glomerulosclerosis and interstitial fibrosis. It was speculated that this difference was associated with the use of immunosuppressive agents. In the present study, the incidence of glomerulosclerosis, tubular atrophy, interstitial fibrosis and arteriosclerosis were compared in the graft loss and functional graft groups. The results showed that glomerulosclerosis, tubular atrophy and interstitial fibrosis were significantly more common in the graft loss group than in the functional graft group. However, the rate of arteriosclerosis was similar in the two groups.

Furthermore, the results of the current study showed no significant difference between the renal graft survival rate in patients with $\operatorname{IgAN}$ and patients with or without other glomerular diseases. It was suggested that these results may be explained by the similar disease severity of the three groups. Concurrent with these results, Kiattisunthorn et al (13) found no significant difference in renal graft survival among these three groups. However, the large population study of Andresdottir et al (21) demonstrated a greater 1-year graft survival rate in renal graft recipients with $\operatorname{IgAN}$ than in those without glomerular diseases. They speculated that the results may be due to the better immune status of patients with IgAN. However, the results also indicated that the graft survival rate tended to decrease more rapidly in renal graft recipients with $\operatorname{Ig} \mathrm{AN}$ than in those without $\operatorname{Ig} \mathrm{AN}$.

In conclusion, renal transplantation is considered the optimum treatment for patients with $\mathrm{IgAN}$-induced ESRD 
as such patients have a high 5- and 10-year graft survival rate even in the presence of recurrent $\operatorname{Ig} \mathrm{AN}$. Following renal transplantation, the presence of numerous prognostic factors indicated poor graft survival, which should result in caution and the implementation of appropriate interventions.

\section{Acknowledgements}

The authors would like to thank Dr Feng Zheng for their input regarding the experimental procedures. This study was supported in part by grants from the Key Laboratory of Fujian Province (grant no. 2010Y2006), the Fujian Natural Science Foundation (grant no. 2012J01404) and the Fujian Science and Technology Project Foundation (grant no. 2012Y5006).

\section{References}

1. Bi ZQ: Clinical and pathological analysis of 40 cases of $\operatorname{Ig} \mathrm{A}$ nephropathy. Zhonghua Nei Ke Za Zhi 23: 136-140, 1984 (In Chinese).

2. Li LS: Clinicopathological correlation of 34 cases of IgA nephropathy. Zhonghua Nei Ke Za Zhi 23: 336-339, 397, 1984 (In Chinese).

3. Berger J, Yaneva H, Nabarra B and Barbanel C: Recurrence of mesangial deposition of IgA after renal transplantation. Kidney Int 7: 232-241, 1975

4. Odum J, Peh CA, Clarkson AR, et al: Recurrent mesangial IgA nephritis following renal transplantation. Nephrol Dial Transplant 9: 309-312, 1994.

5. Kessler M, Hiesse C, Hestin D, et al: Recurrence of immunoglobulin A nephropathy after renal transplantation in the cyclosporin era. Am J Kidney Dis 28: 99-104, 1996.

6. Ohmacht C, Kliem V, Burg M, et al: Recurrent immunoglobulin A nephropathy after renal transplantation: a significant contributor to graft loss. Transplantation 64: 1493-1496, 1997.

7. Mafsugami K, Naito T, Nitta K, et al: A clinicopathological study of recurrent $\operatorname{IgA}$ nephropathy following renal transplantation. Nippon Jinzo Gakkai Shi 40: 322-328, 1998 (In Japanese).

8. Berthoux B, Diconne E, Mariat C, et al: Risk factors for primary IgA nephropathy recurrence after renal transplantation (RTx). Nephrol Dial Transplant 18: 801-802, 2003.

9. Namba Y, Oka K, Moriyama T, et al: Risk factors for graft loss in patients with recurrent IgA nephropathy after renal transplantation. Transplant Proc 36: 1314-1316, 2004.

10. Couser W: Recurrent glomerulonephritis in the renal allograft: an update of selected areas. Exp Clin Transplant 3: 283-288, 2005.

11. Ng YS, Vathsala A, Chew ST, et al: Long term outcome of renal allografts in patients with immunoglobulin A nephropathy. Med J Malaysia 62: 109-113, 2007.
12. Moriyama T, Nitta K, Suzuki K, et al: Latent IgA deposition from donor kidney is the major risk factor for recurrent IgA nephropathy in renal transplantation. Clin Transplant 19: 41-48, 2005.

13. Kiattisunthorn K, Premasathian N, Wongwiwatana A, et al: Evaluating the clinical course and prognostic factors of posttransplantation immunoglobulin A nephropathy. Transplant Proc 40: 2349-2354, 2008.

14. Racusen LC, Solez K, Colvin RB, et al: The Banff 97 working classification of renal allograft pathology. Kidney Int 55: 713-723, 1999.

15. Briganti EM, Russ GR, McNeil JJ, et al: Risk of renal allograft loss from recurrent glomerulonephritis. N Engl J Med 347: 103-109, 2002.

16. Sanfilippo F, Croker BP and Bollinger RR: Fate of four cadaveric donor renal allografts with mesangial IgA deposits. Transplantation 33: 370-376, 1982

17. Ji S, Liu M, Chen J, et al: The fate of glomerular mesangial IgA deposition in the donated kidney after allograft transplantation. Clin Transplant 18: 536-540, 2004.

18. Berger J: Recurrence of IgA nephropathy in renal allografts. Am J Kidney Dis 12: 371-372, 1988.

19. Wang AY, Lai FM, Yu AW, et al: Recurrent IgA nephropathy in renal transplant allografts. Am J Kidney Dis 38: 588-596, 2001.

20. McDonald SP and Russ GR: Recurrence of IgA nephropathy among renal allograft recipients from living donors is greater among those with zero HLA mismatches. Transplantation 82: 759-762, 2006.

21. Soler MJ, Mir M, Rodriguez E, et al: Recurrence of IgA nephropathy and Henoch-Schönlein purpura after kidney transplantation: risk factors and graft survival. Transplant Proc 37: 3705-3709, 2005

22. Andresdottir MB, Haasnoot GW, Doxiadis II, et al: Exclusive characteristics of graft survival and risk factors in recipients with immunoglobulin A nephropathy: a retrospective analysis of registry data. Transplantation 80: 1012-1018, 2005.

23. Andresdottir MB, Haasnoot GW, Persijn GG and Claas FH: HLA-B8, DR3: a new risk factor for graft failure after renal transplantation in patients with underlying immunoglobulin A nephropathy. Clin Transplant 23: 660-665, 2009.

24. Tang Z, Ji SM, Chen DR, et al: Recurrent or de novo IgA nephropathy with crescent formation after renal transplantation. Ren Fail 30: 611-616, 2008.

25. Kowalewska J, Yuan S, Sustento-Reodica N, et al: IgA nephropathy with crescents in kidney transplant recipients. Am J Kidney Dis 45: 167-175, 2005.

26. Oka K, Imai E, Moriyama T, et al: A clinicopathological study of IgA nephropathy in renal transplant recipients: beneficial effect of angiotensin-converting enzyme inhibitor. Nephrol Dial Transplant 15: 689-695, 2000. 